\title{
Long-Term Effects of Bariatric Surgery on Gut Microbiota Composition and Faecal Metabolome Related to Obesity Remission
}

\author{
María Juárez-Fernández ${ }^{1}{ }^{\circledR}$, Sara Román-Sagüillo ${ }^{1}$, David Porras ${ }^{1}{ }^{\circledR}$, María Victoria García-Mediavilla ${ }^{1,2}$, \\ Pedro Linares ${ }^{3}$, María Dolores Ballesteros-Pomar ${ }^{4}{ }^{\circledR}$, Ana Urioste-Fondo ${ }^{4}$, Begoña Álvarez-Cuenllas ${ }^{3}$, \\ Javier González-Gallego ${ }^{1,2} \oplus$, Sonia Sánchez-Campos ${ }^{1,2} \oplus$, Francisco Jorquera ${ }^{1,2,3}$ and Esther Nistal ${ }^{1,2, *}$ \\ 1 Instituto Universitario de Biomedicina (IBIOMED), Universidad de León, 24007 León, Spain; \\ mjuarf@unileon.es (M.J.-F.); sroms@unileon.es (S.R.-S.); dpors@unileon.es (D.P.); \\ mvgarm@unileon.es (M.V.G.-M.); jgonga@unileon.es (J.G.-G.); ssanc@unileon.es (S.S.-C.); \\ fjorqueraplaza@gmail.com (F.J.) \\ 2 Centro de Investigación Biomédica en Red de Enfermedades Hepáticas y Digestivas (CIBERehd), \\ Instituto de Salud Carlos III, 28029 Madrid, Spain \\ 3 Servicio de Aparato Digestivo, Complejo Asistencial Universitario de León, 24071 León, Spain; \\ plinares68@gmail.com (P.L.); bealvcue@gmail.com (B.Á.-C.) \\ check for \\ updates \\ Citation: Juárez-Fernández, M.; \\ 4 Departamento de Endocrinología y Nutrición, Complejo Asistencial Universitario de León, 24071 León, Spain; \\ mdballesteros@telefonica.net (M.D.B.-P.); anaurifon@gmail.com (A.U.-F.) \\ * Correspondence: menisg@unileon.com; Tel.: +34-9-8729-1997
} Román-Sagüillo, S.; Porras, D.; García-Mediavilla, M.V.; Linares, P.; Ballesteros-Pomar, M.D.; UriosteFondo, A.; Álvarez-Cuenllas, B.; González-Gallego, J.; SánchezCampos, S.; et al. Long-Term Effects of Bariatric Surgery on Gut Microbiota Composition and Faecal Metabolome Related to Obesity Remission. Nutrients 2021, 13, 2519. https://doi.org/10.3390/nu13082519

Academic Editors: Margarita Aguilera and Mohsen Mazidi

Received: 10 June 2021

Accepted: 19 July 2021

Published: 23 July 2021

Publisher's Note: MDPI stays neutral with regard to jurisdictional claims in published maps and institutional affiliations.

Copyright: (C) 2021 by the authors Licensee MDPI, Basel, Switzerland This article is an open access article distributed under the terms and conditions of the Creative Commons Attribution (CC BY) license (https:/ creativecommons.org/licenses/by/ $4.0 /)$

\begin{abstract}
Obesity is one of the main worldwide public health concerns whose clinical management demands new therapeutic approaches. Bariatric surgery is the most efficient treatment when other therapies have previously failed. Due to the role of gut microbiota in obesity development, the knowledge of the link between bariatric surgery and gut microbiota could elucidate new mechanistic approaches. This study aims to evaluate the long-term effects of bariatric surgery in the faecal metagenome and metabolome of patients with severe obesity. Faecal and blood samples were collected before and four years after the intervention from patients with severe obesity. Biochemical, metagenomic and metabolomic analyses were performed and faecal short-chain fatty acids were measured. Bariatric surgery improved the obesity-related status of patients and significantly reshaped gut microbiota composition. Moreover, this procedure was associated with a specific metabolome profile characterized by a reduction in energetic and amino acid metabolism. Acetate, butyrate and propionate showed a significant reduction with bariatric surgery. Finally, correlation analysis suggested the existence of a long-term compositional and functional gut microbiota profile associated with the intervention. In conclusion, bariatric surgery triggered long-lasting effects on gut microbiota composition and faecal metabolome that could be associated with the remission of obesity.
\end{abstract}

Keywords: bariatric surgery; gut microbiota; metabolomics; metagenomics; obesity; short-chain fatty acids

\section{Introduction}

Obesity is one of the main public concerns worldwide, linked to increased rates of morbidity and mortality besides high resources demanding for public health systems [1] Clinical management of overweight and obesity is traditionally based on lifestyle interventions due to the limited availability and efficacy of pharmacological therapies [2]. Bariatric surgery is considered the gold standard treatment when nonsurgical alternatives have failed, with great performance in remedying pathology in the short and in the long term $[3,4]$. Benefits of bariatric surgery exceed simple weight loss, counteracting other features of the metabolic dysfunction associated with obesity, including type 2 diabetes mellitus or hypertension [5]. 
The preponderant role of intestinal microbiota in the development of obesity is accepted in the current knowledge of the disease [6]. Under this perspective, the capacity of bariatric surgery to reshape gut microbiota as one of the mechanisms underlying its therapeutic success has been proposed, and several findings have been reported in this sense. For example, a greater microbial gene richness and bacterial diversity, features associated to a healthy microbiota, have been observed after bariatric surgery, although great interstudy and interindividual variability regarding changes in specific microbial taxa have been argued [7]. Moreover, the causal role of bariatric surgery-mediated changes in gut microbiota composition in its antiobesity effect has been demonstrated by reduced fat mass gain in germ-free mice colonized with faecal microbiota from operated donors [8].

One of the main contributions of the gut microbiota to the instauration of obesity resides in energy extraction from dietary nutrients. The microbiota of individuals with obesity is believed to have increased capacity for energy harvest [9], thus providing larger amounts of energetic metabolites such as short chain fatty acids (SCFAs) from plant polysaccharides [10]. Additionally, microbial metabolism of dietary nutrients may produce potential harmful substances related to the instauration of the low-grade inflammatory state of obesity [11]. Weight loss by means of dietary interventions modify gut microbiota, counteracting the extensively reported elevated Firmicutes/Bacteroidetes ratio in obesity and increasing beneficial Verrucomicrobia phylum [12], but also promoting functional changes in the microbiota and altering its derived metabolites [13,14]. Likewise, bariatric surgery could drive a shift of the metabolic capacity of the gut microbiota towards a lean-like phenotype, both from a composition and from a functional point of view, related to the good outcomes of the procedure.

Here we report the results of a longitudinal long-term study with a multiomics approach to characterise the impact of bariatric surgery on gut microbiota and associated metabolome in patients with clinically severe obesity.

\section{Materials and Methods}

\subsection{Study Population}

Nine patients with severe obesity were included in the study to analyze their status before and four years after bariatric surgery. All patients were characterized in detail from a demographic point of view (age, sex and race). The age range of the patients was between 20 and 60 years old, with two men and seven women, all of them Caucasians. They were also characterized from an anthropometric and biochemical point of view. Likewise, insulin resistance was determined using the homeostatic model assessment (HOMA-IR). None of the patients had been treated with antibiotics for at least one month prior to sampling.

The surgical procedures were laparoscopic vertical sleeve gastrectomy (VSG) $(n=6)$, biliopancreatic diversion (BPD) $(n=2)$ and gastric bypass $(n=1)$, selected according to the protocols of the High-Risk Obesity Unit from Complejo Asistencial Universitario of León (CAULE). Patient evolution was monitored by the Endocrinology and Digestive Diseases Services of the CAULE, evaluating the changes derived from the surgical intervention in the previously indicated parameters. Moreover, after surgery, patients underwent nutritional education by a registered dietitian. The study was conducted according to the guidelines outlined in the Declaration of Helsinki, and all procedures involving human subjects were approved by the Ethics Committee of the Complejo Asistencial Universitario de León (CAULE) (protocol code 2135, 23 February 2021). All patients gave informed written consent before participating in the study.

\subsection{Sample Collection}

Blood samples of patients with severe obesity before and four years after bariatric surgery were collected to determine the above-mentioned biochemical parameters (Figure 1). After the intervention, a blood sample from one patient could not be collected, as illustrated in Figure 1. 


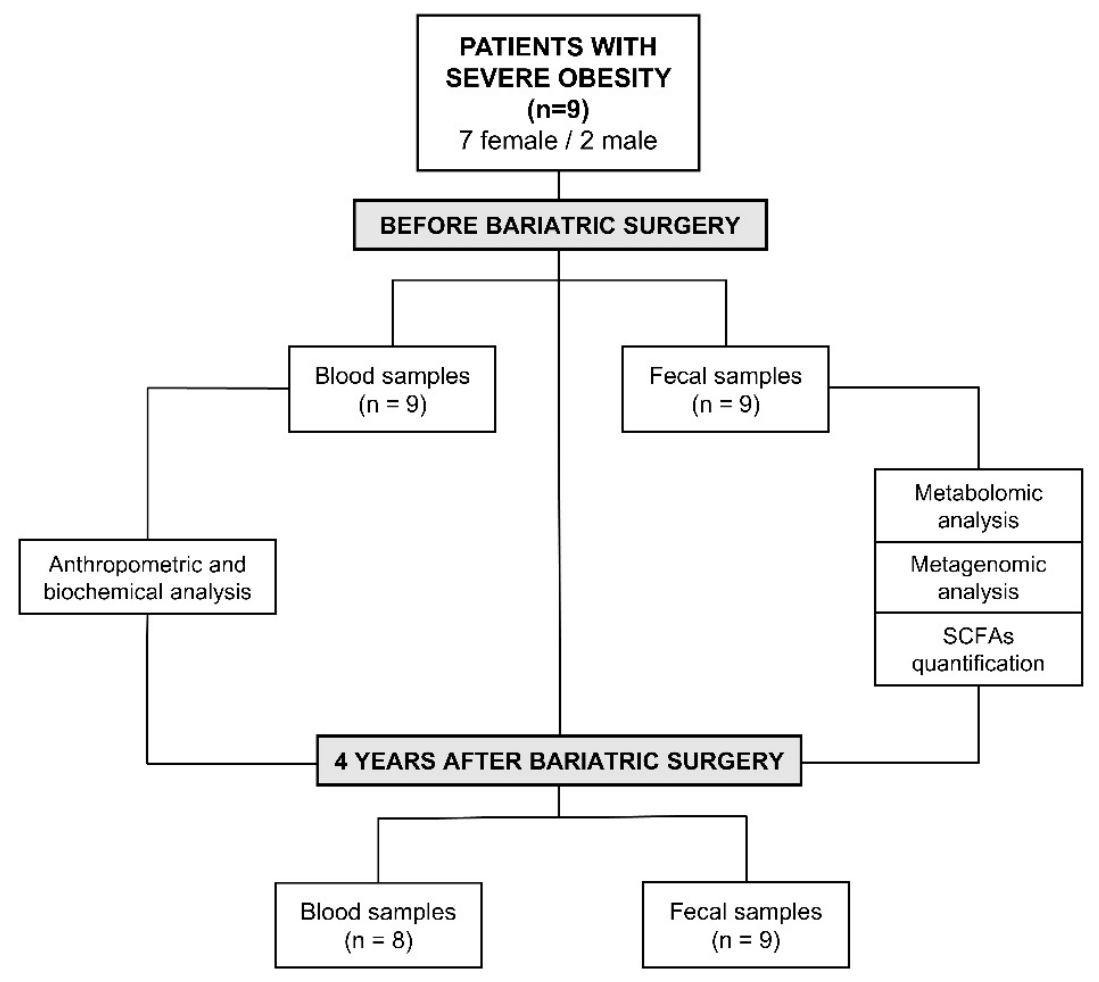

Figure 1. Experimental design and characteristics of the patients included in the study.

Moreover, fresh stool samples were collected before and after surgical intervention from the nine patients included in the study (Figure 1). All samples were homogenized and aliquoted within three hours of defecation. The aliquots were stored at $-80{ }^{\circ} \mathrm{C}$ until analysis.

\subsection{Faecal Metagenomic Analysis}

Total genomic DNA was isolated from faecal samples using the Qiagen Fast DNA Stool Mini Kit (Qiagen, Hilden, Germany) according to manufacturer instructions with some modifications previously described [15]. A mechanical lysis step was included based on an initial bead-beating step for $10 \mathrm{~min}$, and lysis temperature was increased to $95^{\circ} \mathrm{C}$ to facilitate DNA extraction from Gram positive bacteria. DNA concentration was determined by using a NanoDrop-1000 spectrophotometer (NanoDrop Technologies, Wilmington, DE, USA). DNA samples were stored at $-20^{\circ} \mathrm{C}$ until their analysis.

Amplification of the $16 \mathrm{~S}$ rRNA V3-V4 hypervariable region was carried out by PCR as previously described [16]. Triplicate PCR assays for each sample were performed and purified with the Wizard ${ }^{\circledR}$ Genomic DNA Purification kit (Promega, Madison, WI, USA) following the manufacturer's instructions.

The resulting amplicons were cleaned, quantified and sequenced on an Illumina MiSeq platform. The Illumina bcl2fastq $\odot$ program was used to demultiplex sequencing data. Fastp and FastQC v0.11.8 [17] tools were used to check for quality, adapter trimmed and filtered forward and reversing raw reads. Quantitative Insights into Microbial Ecology software (QIIME version 1.9.1 [18]) was used, including forward and reverse reads joining, chimera removal, data filtering and taxonomic annotation. To remove chimeric sequences from the reads, the Usearch6.1 algorithm was used [19]. These sequences were clustered into operational taxonomic units (OTUs) using UCLUST, with a similarity threshold of 0.97 [20], and were aligned using PyNast against the 16S reference database GreenGenes (version 13.8) [21] using default parameters [20]. 


\subsection{Faecal Metabolomic Analysis}

Sample analysis was carried out by MS-Omics as follows. The analysis was performed using a Thermo Scientific Vanquish LC coupled to a ThermoQ Exactive HF-MS. An electrospray ionization interface was used as the ionization source. Analysis was performed in negative and positive ionization modes. The UPLC was performed using a slightly modified version of the protocol described by Catalin et al. (UPLC/MS Monitoring of Water-Soluble Vitamin Bs in Cell Culture Media in Minutes, Water Application note 2011, 720004042en). Peak areas were extracted using Compound Discoverer 2.0.

\subsection{SCFAs Identification and Quantification}

Sample analysis was carried out by MS-Omics as follows. Samples were acidified using hydrochloric acid, and deuterium-labelled internal standards were added. All samples were analysed in a randomized order. Analysis was performed using a high polarity column (Zebron ${ }^{\mathrm{TM}}$ ZB-FFAP, GC Cap. Column $30 \mathrm{~m} \times 0.25 \mathrm{~mm} \times 0.25 \mu \mathrm{m}$ ) installed in a GC (7890B, Agilent, Wilmington, DE, USA) coupled with a quadropole detector (5977B, Agilent). The system was controlled by ChemStation (Agilent). Raw data was converted to netCDF format using Chemstation (Agilent), before the data was imported and processed in Matlab R2014b (Mathworks, Inc., Natick, MA, USA) using the PARADISe software earlier described [22].

\subsection{Statistical Analysis}

Student's t test was employed to evaluate significant differences and $p<0.05$ was considered significant for differences. From microbiota composition and metabolomic data, statistical significance was determined by the nonparametric Wilcoxon test with $p<0.05$. A false discovery rate (FDR)-adjusted $p<0.05$ was considered for significance. Statistical analyses were performed using SPSS 24.0 software for Windows (Chicago, IL, USA) and R software (R-project, Vienna, Austria).

\section{Results}

3.1. Effect of Bariatric Surgery on Biochemical and Anthropometric Clinical Characteristics of Patients with Severe Obesity

The characteristics and clinical data, as well as the evolution in body weight loss of the participants of the study before and after bariatric surgery are depicted in Table 1 and Table S1.

Bariatric technique enhanced biochemical and anthropometric parameters compared with measurements before surgery, showing statistical differences between patients with obesity before and after the intervention in the main parameters associated with obesity and metabolic syndrome. These included body weight and body mass index (BMI), body fat percentage, glucose, insulin and HOMA-IR, percentage of glycated haemoglobin (HbA1c), high density lipoprotein cholesterol (HDL), uric acid, aspartate transaminase (AST) levels and $C$ reactive protein $(\mathrm{CRP})$ concentration $(p<0.05)$ (Table 1$)$. Moreover, the percentage of weight loss of our patients after four years of bariatric surgery was $32.13 \pm 2.46 \%$, with a body weight recovery of $6.10 \pm 2.84 \%$, compared with the point of maximum body weight loss, which was the second year after the intervention (Table S1). 
Table 1. Anthropometric and biochemical information before and after bariatric surgery of patients with severe obesity.

\begin{tabular}{|c|c|c|c|}
\hline & Pre BS & Post BS & $p$ Value \\
\hline \multicolumn{4}{|c|}{ Anthropometric parameters } \\
\hline Weight (kg) & $124.98 \pm 6.33$ & $85.78 \pm 5.81^{* * *}$ & $1 \times 10^{-6}$ \\
\hline BMI $\left(\mathrm{kg} / \mathrm{m}^{2}\right)$ & $45.46 \pm 2.05$ & $31.09 \pm 1.81 * * *$ & $4 \times 10^{-6}$ \\
\hline Body fat (\%) & $50.75 \pm 1.03$ & $36.54 \pm 2.91 * *$ & 0.003 \\
\hline Waist circumference $(\mathrm{cm})$ & $130.63 \pm 4.54$ & $111.71 \pm 7.05$ & 0.059 \\
\hline Hip circumference $(\mathrm{cm})$ & $131.13 \pm 8.83$ & $102.17 \pm 8.26^{* *}$ & 0.008 \\
\hline Systolic blood pressure (mm) & $133.63 \pm 3.23$ & $118 \pm 6.77$ & 0.064 \\
\hline Diastolic blood pressure (mm) & $87.38 \pm 2.52$ & $86.86 \pm 5.82$ & 0.966 \\
\hline \multicolumn{4}{|c|}{ Biochemical parameters } \\
\hline Glucose (mg/dL) & $91 \pm 3.08$ & $83 \pm 2.49^{* *}$ & 0.009 \\
\hline Insulin $(\mathrm{mUI} / \mathrm{mL})$ & $21.46 \pm 3.12$ & $7.08 \pm 1.05^{* *}$ & 0.001 \\
\hline HOMA-IR & $4.34 \pm 0.59$ & $1.45 \pm 0.21 * *$ & 0.001 \\
\hline $\mathrm{HbA1c}(\%)$ & $5.76 \pm 0.13$ & $5.19 \pm 0.15^{* *}$ & 0.009 \\
\hline Total cholesterol (mg/dL) & $175.75 \pm 6.39$ & $161.88 \pm 14.84$ & 0.427 \\
\hline LDL-cholesterol (mg/dL) & $105.5 \pm 6.65$ & $80.38 \pm 11.35$ & 0.120 \\
\hline HDL-cholesterol (mg/dL) & $51.50 \pm 3.01$ & $66.5 \pm 4.07^{* *}$ & 0.004 \\
\hline Triglycerides (mg/dL) & $93.75 \pm 13.67$ & $73.38 \pm 11.95$ & 0.247 \\
\hline Uric acid (mg/dL) & $5.68 \pm 0.42$ & $4.37 \pm 0.34$ * & 0.033 \\
\hline Urea $(\mathrm{mg} / \mathrm{dL})$ & $30.75 \pm 2.70$ & $31.75 \pm 2.66$ & 0.584 \\
\hline Creatinine $(\mathrm{mg} / \mathrm{dL})$ & $0.72 \pm 0.03$ & $0.72 \pm 0.07$ & 0.933 \\
\hline AST $(\mathrm{U} / \mathrm{L})$ & $19.38 \pm 1.44$ & $14.88 \pm 1.11$ * & 0.013 \\
\hline $\operatorname{ALT}(\mathrm{U} / \mathrm{L})$ & $25.75 \pm 3.60$ & $20.75 \pm 8.29$ & 0.476 \\
\hline GGT (U/L) & $31.38 \pm 6.42$ & $16.5 \pm 4.57$ & 0.059 \\
\hline Vitamin D (ng/mL) & $27.25 \pm 2.96$ & $52.25 \pm 10.58$ & 0.063 \\
\hline $\mathrm{CRP}(\mathrm{mg} / \mathrm{L})$ & $19.63 \pm 10.98$ & $2.71 \pm 1.37$ * & 0.017 \\
\hline
\end{tabular}

Data are presented as mean \pm SEM. Pre BS, pre bariatric surgery; Post BS, post bariatric surgery; BMI, body mass index; HOMA-IR, homeostatic model assessment for insulin resistance; LDL, low density lipoprotein; HDL, high density lipoprotein; AST, aspartate transaminase; ALT, alanine transaminase; GGT, gamma-glutamyl transferase $\mathrm{CRP}, \mathrm{C}$ reactive protein. ${ }^{*} p<0.05 ;{ }^{* *} p<0.01 ;{ }^{* *} p<0.001$ vs. patients with obesity before bariatric surgery (Pre BS) by Student's $t$-test.

\subsection{Differences in Faecal Microbiota Composition Associated to Bariatric Surgery}

A cladogram was performed to show the main differences in gut microbiota composition before and after bariatric surgery based on the linear discriminant analysis (LDA) effect size (LEfSe) allowing us to identify which taxa were contributing to discrimination between groups (Figure 2A and Figure S1A). Eleven and 13 bacterial taxa enriched from patients with severe obesity before and after surgery, respectively, were identified. Enriched phylotypes from obese patients before intervention were predominantly from Cyanobacteria phyla and the Clostridia class, whereas most from obese patients after surgery were enriched principally from the Coriobacteriia class. 
A
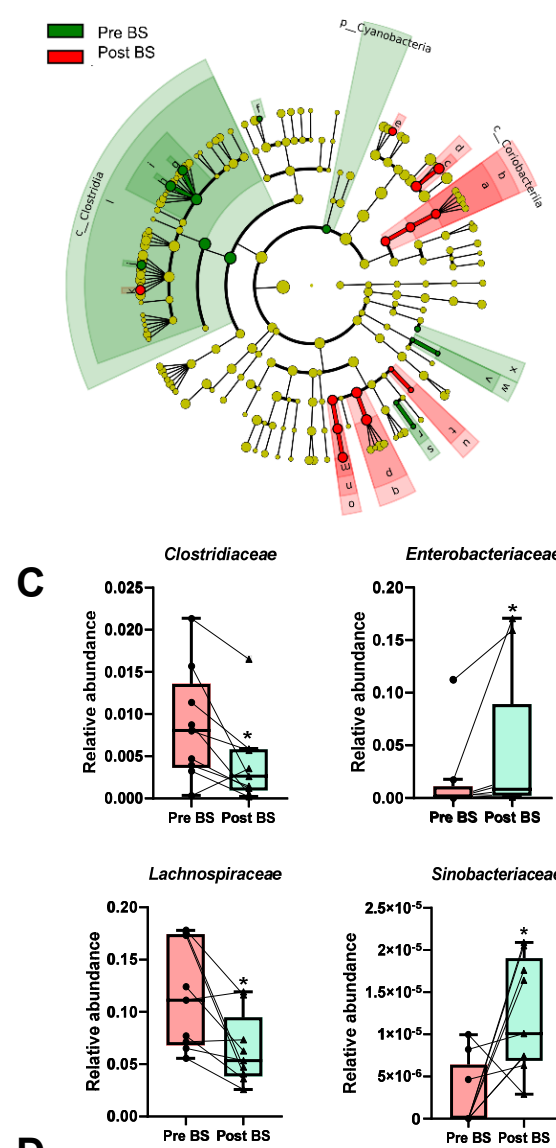

D
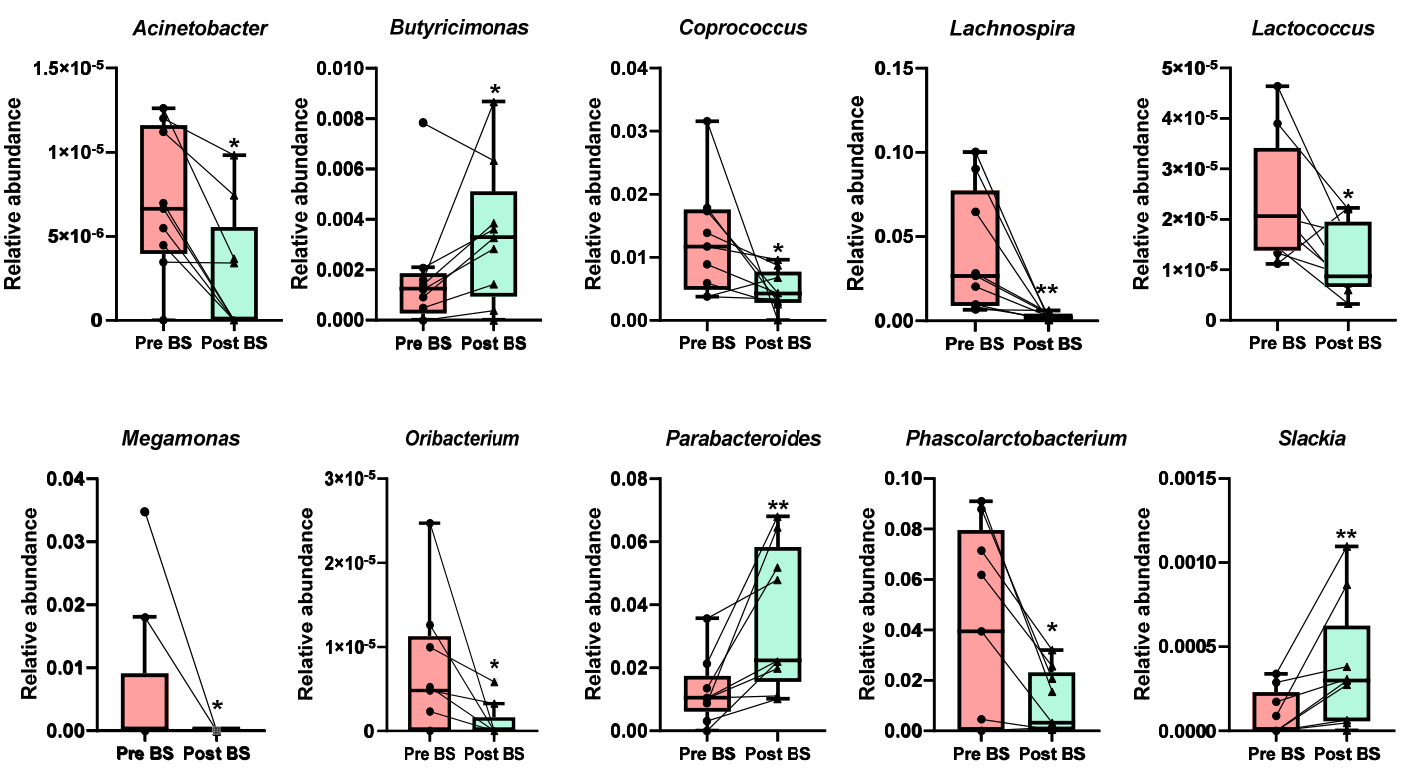

Figure 2. Effect of bariatric surgery on gut microbiota composition of patients with severe obesity. (A) Cladogram showing the main differences in gut microbiota composition before (Pre BS) and after (Post BS) bariatric surgery based on linear discriminant analysis effect size (LEfSe). Colour denotes taxa enriched in Pre BS samples (red) and Post BS samples (green). A cut-off value of LDA $>2$ and a Wilcoxon $p$ value $<0.05$ were set up to identify discriminating taxa. The LEfSe results plot is provided in Figure S1. (B) Relative abundance of phyla in the patients with severe obesity Pre BS and Post BS. (C) Differences in the relative abundance at class level of the patients with severe obesity Pre BS and Post BS. (D) Differences in the relative abundance at genus level of the patients with severe obesity Pre BS and Post BS. Nonparametric Wilcoxon signed-rank test. ${ }^{*} p<0.05 ; * * 0.01$ vs. Pre BS. 
A principal coordinate analysis (PCoA) based on the Morisita Horn index was performed to analyse the influence of e bariatric surgery on bacterial community distribution at the OTU level. This analysis revealed a clear separation of the faecal microbiota according to the second axis based on the intervention, which accounted for $15.25 \%$ of the total variance (Figure S1B). Additionally, bariatric surgery tended to increase gut microbiota diversity as calculated by the Shannon index, though these differences were not significant (Figure S1C).

Bariatric surgery transformed gut microbiota composition at different taxonomic levels, highlighting a significant reduction in Firmicutes phylum in comparison to the same patients before the intervention, whereas, the relative abundance of Proteobacteria and Lentisphaerae phyla was significantly increased in response to bariatric procedure (Figure 2B). Changes were also detected at the family level, in that Enterobacteriaceae and Sinobacteriaceae were significantly increased in operated patients. On the contrary, Clostridiaceae and Lachnospiraceae families were reduced in post bariatric surgery patients (Figure 2C). The most remarkable differences were observed at the genus level. Interestingly, a higher detection of Butyricimonas, Parabacteroides and Slackia genera was observed after the intervention, while genera such as Acinetobacter, Coprococcus, Lachnospira, Lactococcus, Megamonas, Oribacterium and Phascolarctobacterium, which dominated in nonoperated obese patients, were significantly decrease after the surgical procedure (Figure 2D).

\subsection{Effect of Bariatric Surgery on Faecal Metabolomic Profile of Patients with Severe Obesity}

The Partial Least-Squares Discriminant Analysis (PLS-DA) of faecal metabolites showed a clear separation of the patients with severe obesity before and after bariatric surgery (accuracy 0.88889; R2 0.99074; Q2 0.5581), suggesting that the surgical intervention modified their faecal metabolome significantly (Figure 3A). The metabolites which allowed discrimination between both groups in the PLS-DA are included in Figure S2A as a Variable Importance in Projection (VIP) scores plot. Moreover, the individual representation of the normalized abundance of the top 25 discriminating faecal metabolites of each patient before and after the intervention in a heatmap showed a clear and different metabolomic profile depending on the bariatric surgery (Figure 3B).

To identify the metabolites that changed the most with bariatric surgery, a Volcano plot based on the Wilcoxon signed-rank test $(p<0.05$, fold-change $(\mathrm{FC})>2)$ was performed, in which a total of 35 metabolites were identified to significantly change because of the intervention. As shown in Figure 3C, the abundance of methyl acetoacetate, carbamoyl aspartate and serine phosphate were significantly reduced with bariatric surgery, whereas other metabolites, such as taurine or tropic acid, were increased with the intervention. Bariatric surgery also diminished significantly the faecal concentration of 5-aminolevulinic acid, $\gamma$-glutamylvaline, acetamide, citric acid, glyoxylic acid, malic acid, maltotriose, N8acetylspermidine, protocatechuic acid, trimethylamine- $\mathrm{N}$-oxide (TMAO), valeronitrile and vanillin, among others (Figure S2B).

Furthermore, a pathway enrichment analysis based on the faecal metabolites detected in the patients of study was performed to identify the metabolic pathways which significantly changed due to bariatric surgery (Figure 3D). The tricarboxylic acid (TCA) cycle, glycine, serine and threonine metabolism, glyoxylate and dicarboxylate metabolism, tyrosine metabolism and the alanine, aspartate and glutamate metabolism (Figure 3D), were the pathways on which the bariatric surgery had the biggest impact. 
A

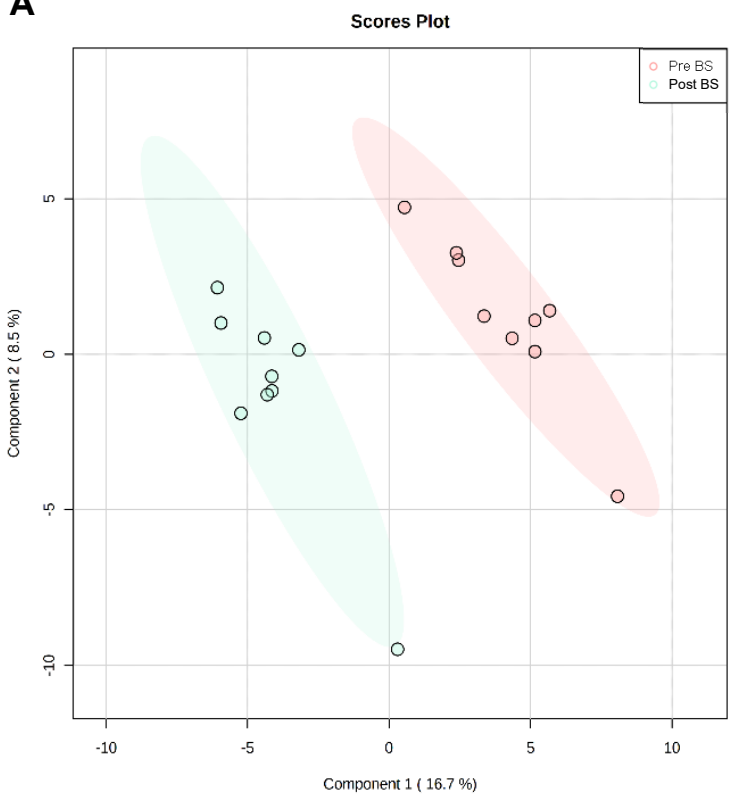

C

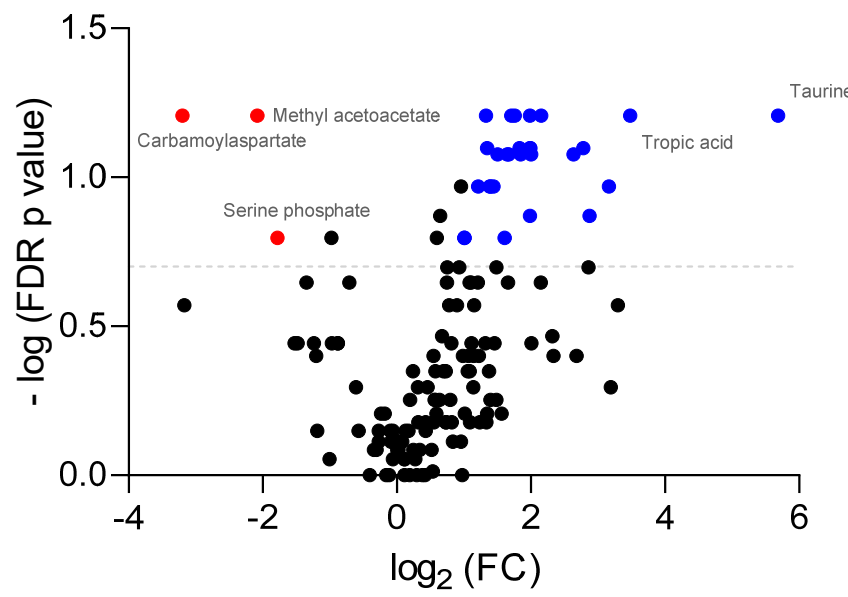

B

D
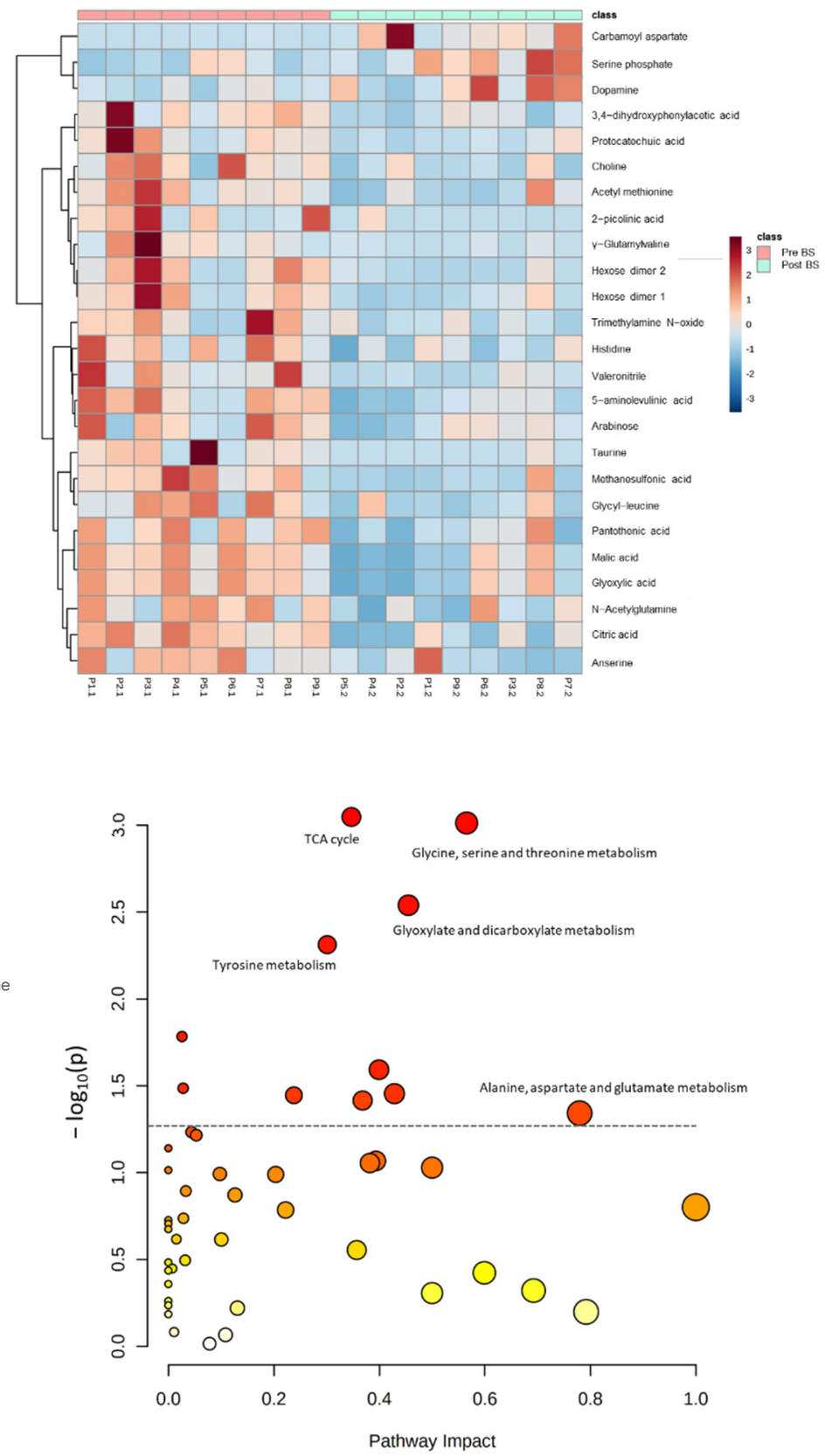

Figure 3. Effect of bariatric surgery on faecal metabolome of patients with severe obesity. (A) Partial Least-Squares Discriminant Analysis (PLS-DA) of faecal metabolites of patients with severe obesity before (Pre BS) and after (Post BS) bariatric surgery. (B) Heatmap showing normalized abundance of the top 25 discriminating faecal metabolites based on univariate $t$ tests in the patients with severe obesity Pre BS and Post BS. Colour scale denotes higher (red) to lower (blue) abundance. Left side dendrogram represents correlations between metabolites based on Pearson's correlation. (C) Volcano plot showing faecal metabolites with Wilcoxon signed-rank test $p<0.05$ and fold-change (FC) $>2$ (positive FC in blue, negative FC change in red) due to bariatric surgery. (D) Pathway enrichment analysis showing the metabolic pathways significantly associated with surgery. The circle colour (from light yellow to red) is based on its $p$-value, and radius represents pathway impact value. Discontinue grey line denotes $p$ value threshold of 0.05 . 


\subsection{SCFAs Faecal Profile before and after Bariatric Surgery of Patients with Severe Obesity}

The SCFAs faecal profile of the patients was analysed by gas chromatography-mass spectrometry (GC-MS) to determine whether bariatric surgery had modified these molecules. As illustrated in Figure 4A, Principal Component Analysis (PCA) showed that the patients before bariatric surgery were grouped separately from patients after bariatric surgery according to the first axis, with accounted for $57.1 \%$ of the total variance. Among the SCFAs identified, the concentrations of acetate, butyrate and propionate were significantly reduced after bariatric surgery (Figure 4B).

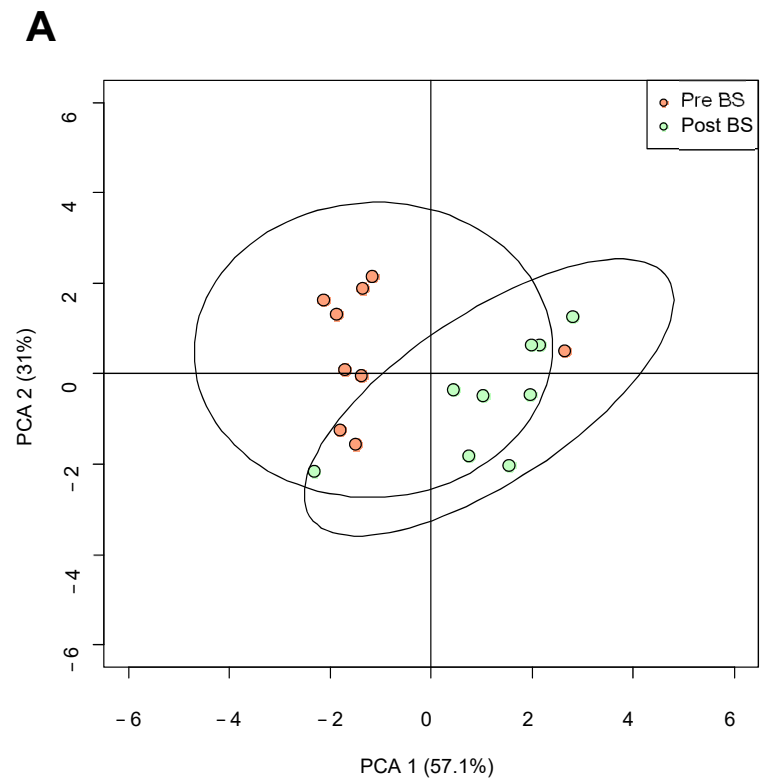

B

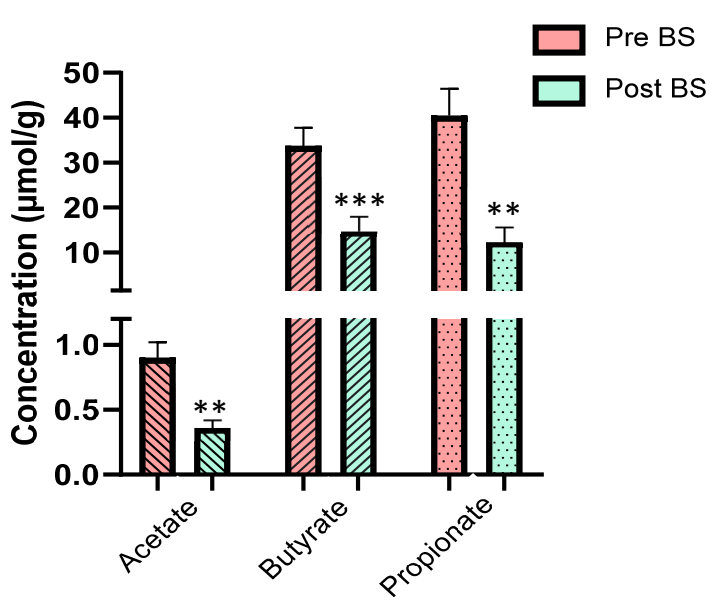

C
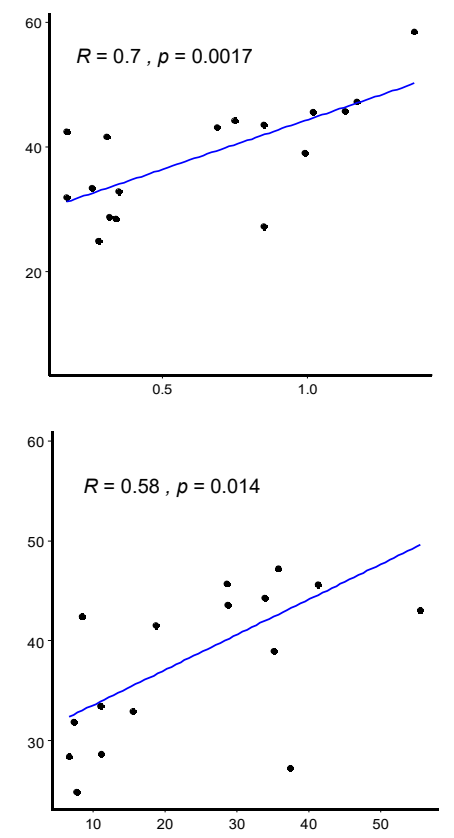

D

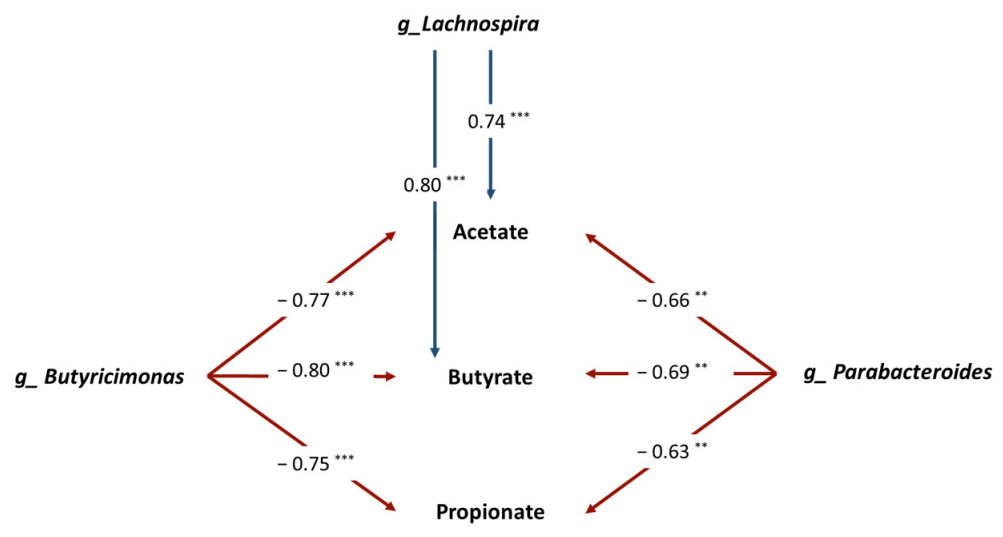

Figure 4. Faecal short-chain fatty acids profile before and after bariatric surgery in patients with severe obesity. (A) Principal coordinate analysis (PCA) showing the differences in the faecal short-chain fatty acids profile of patients before (Pre BS) and after (Post BS) bariatric surgery. (B) Differences in the faecal concentration ( $\mu \mathrm{mol} / \mathrm{g}$ of faeces) of the main short-chain fatty acids in patients with severe obesity Pre BS and Post BS. Nonparametric Wilcoxon signed-rank test. ${ }^{* *} p<0.01{ }^{* * *} p<0.01$ vs. Pre BS. (C) Pearson's correlation coefficients and linear relationships of the main short-chain fatty acids (acetate, butyrate and propionate) and the biochemical and anthropometric parameters. (D) Associations of gut microbiota composition with the main short-chain fatty acids (acetate, butyrate and propionate). The values in arrows represent Spearman's correlation coefficients of each pair of parameters. ${ }^{* *} p<0.01 ;{ }^{* * *} p<0.01$. Detailed XY representation of each association is provided in Figure S3. 


\subsection{Correlations between Biochemical and Anthropometric Parameters, Gut Microbiota Composition and Metabolomic Profile of Patients with Severe Obesity}

Correlation analysis was performed to identify possible links between the benefits and improvements of bariatric surgery and the faecal SCFAs, metabolomic and metagenomic profiles.

Related to the faecal profile of SCFAs, Pearson' correlation analysis between biochemical and anthropometric parameters that changed with bariatric surgery and the main SCFAs showed that acetate, butyrate and propionate significantly and positively correlated with body mass index (BMI), suggesting that the decrease in these metabolites is involved in the improvement of obesity. Moreover, insulin plasma levels also positively correlated with acetate concentration. (Figure 4C). Additionally, Spearman' correlation analysis between SCFAs and gut microbiota composition at the genus level was performed. As shown in Figure 4D, the genera Butyricimonas and Parabacteroides negatively correlated with acetate, butyrate and propionate, whereas Lachnospira correlated positively. The detailed XY representation of each correlation is provided in Figure S3.

Moreover, correlation analysis of biochemical and anthropometric parameters, gut microbiota composition and faecal metabolites after bariatric surgery were performed using Spearman's correlation (Figure 5). From a global point of view, bariatric surgery was associated with a specific metagenomic and metabolomic profile, which could be linked with the beneficial effects on biochemical and anthropometric parameters observed after the intervention. Spearman's correlation between biochemical and anthropometric parameters and faecal metabolites highlighted that citric acid was positively correlated with $\mathrm{HbA1c}$, BMI, AST and ALT, whereas carbamoyl aspartate showed an opposite pattern (Figure 5A). Furthermore, correlation analysis between biochemical and anthropometric parameters and gut microbiota taxa (Figure 5B) showed that the Lachnospira genus positively correlated with $\mathrm{HbA1c}$, BMI, body fat and insulin while Parabacteroides and parameters like $\mathrm{HbA} 1 \mathrm{c}$, uric acid and BMI were negatively correlated. Finally, the heatmap performed between gut microbiota and faecal metabolites (Figure 5C) showed that Lachnospira positively correlated with different metabolites such as TMAO, carbamoyl aspartate, hexose dimers 1 and 2 and 5-aminolevulinic acid. In contrast, Butyricimonas correlated negatively with these metabolites, as well as with tropic acid and valeronitrile. 

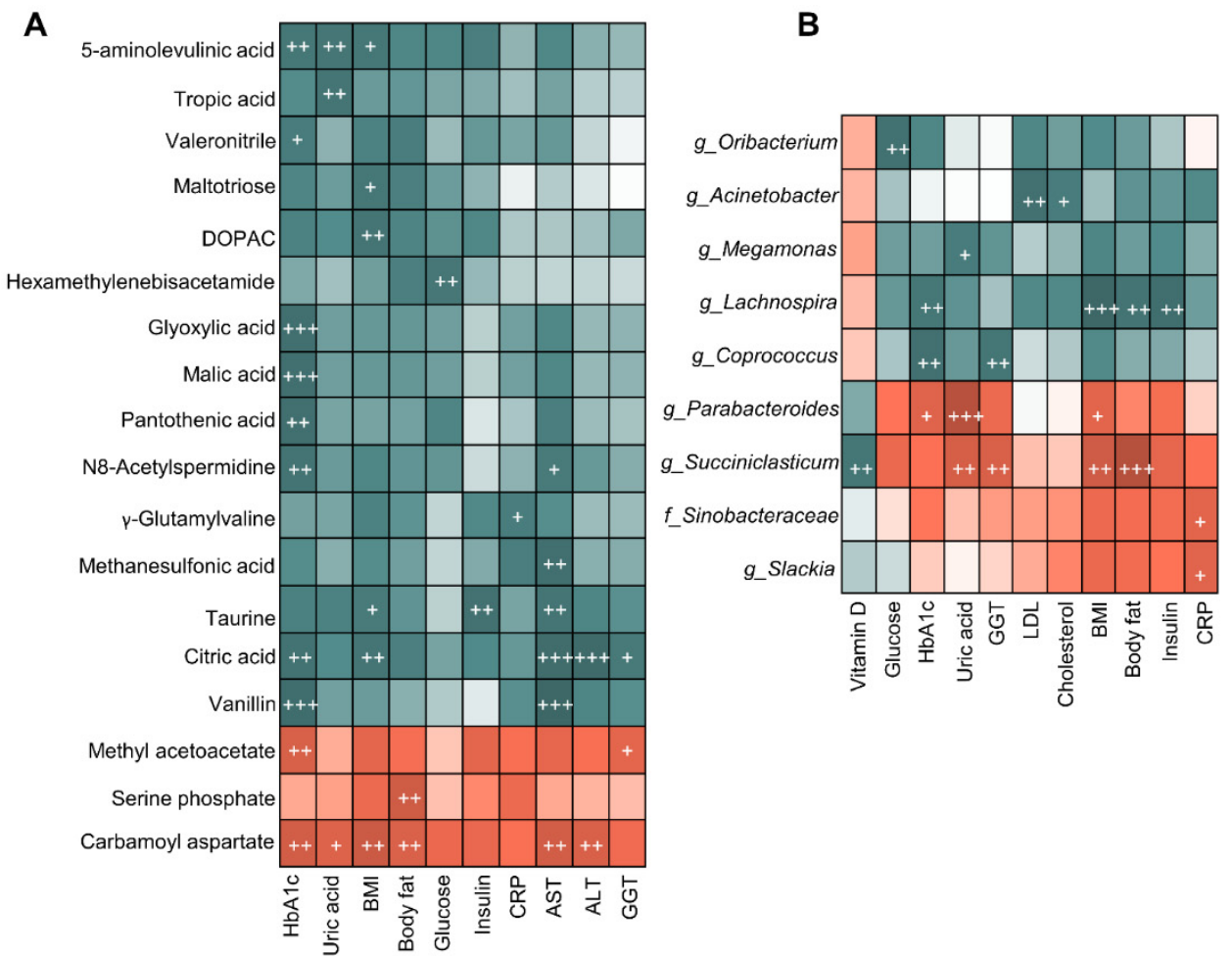

C
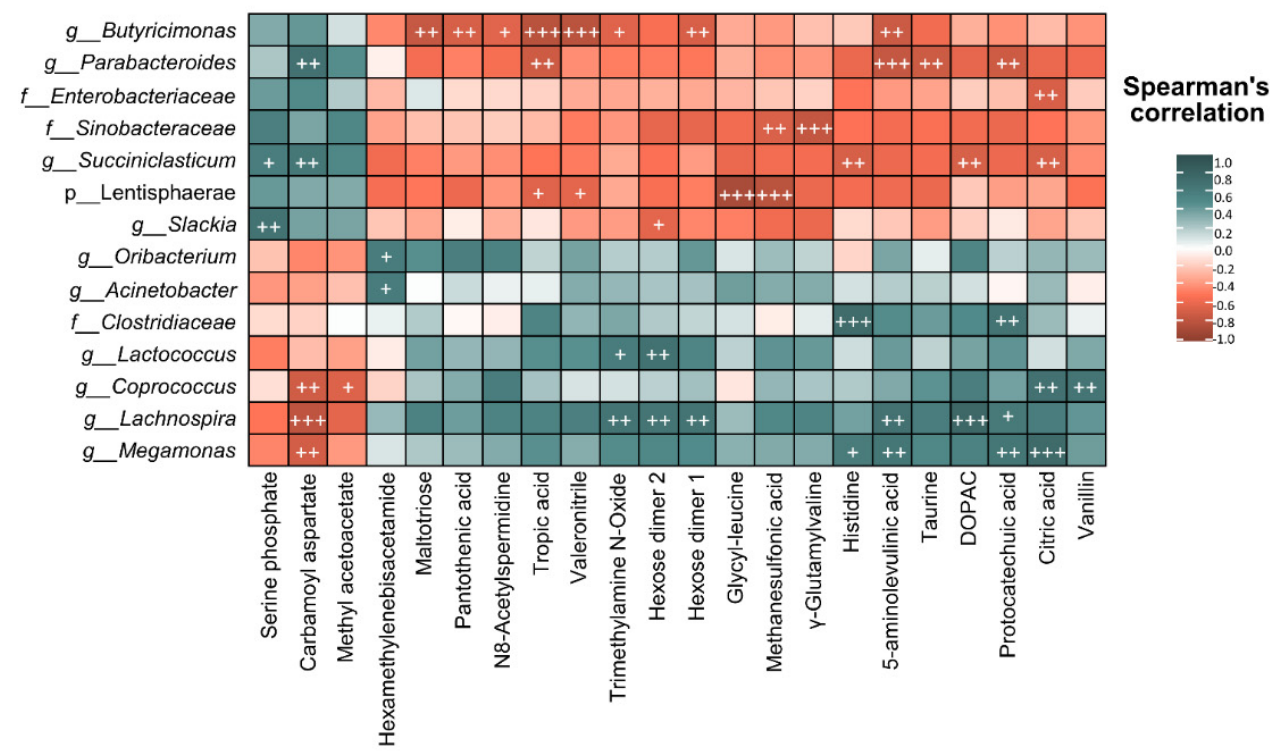

Figure 5. Correlation analysis between biochemical and anthropometric parameters, microbiota composition and the metabolomic profile of patients with severe obesity under bariatric surgery. (A) Heatmap of correlations between faecal metabolomic profile and faecal microbiota composition considering results of patients with severe obesity before and after bariatric surgery. (B) Heatmap of correlations between faecal metabolomic profile and biochemical and anthropometric parameters considering results of patients with severe obesity before and after bariatric surgery. (C) Heatmap of correlations between faecal microbiota composition and biochemical and anthropometric parameters considering results of patients with severe obesity before and after bariatric surgery. Each square represents the Spearman's correlation coefficient $(p<0.01)$. Red and green cells designate positive and negative correlations, respectively. White crosses denote level of significance ${ }^{+} p<0.01,{ }^{++} p<0.005,{ }^{+++} p<0.001$. 


\section{Discussion}

In this study, we developed a longitudinal long-term analysis in which the faecal metabolome and metagenome of patients with severe obesity were determined before and after bariatric surgery to characterize the persistent effects of the intervention. First, our results showed that bariatric surgery had a profound effect on biochemical and anthropometric parameters, improving the health status of the patients four years after the intervention, as was previously described [23]. In this sense, bariatric surgery had a huge effect in body weight loss, ranging from $25-40 \%$ [24], findings that agree with the percentage of weight loss observed at the fourth year in our patients. Nevertheless, weight gain is a common complication after the intervention, with approximately one third of patients regaining excessive weight, which is defined as $\geq 25 \%$ of total lost weight [25]. In our study, a body weight regain of $6.10 \%$ at the fourth year was observed in the patients, a result which cannot be considered a remarkable regain.

Bariatric surgery not only has effects on clinical status, but also modulatory and reshaping effects on gut microbiota. Our results showed that bariatric surgery modified the gut microbiota profile of the patients with severe obesity, as previously reported [26-29]. At the phylum level, bariatric surgery reduced the abundance of Firmicutes phylum, whereas Proteobacteria showed an opposite pattern, reinforcing previous studies [30,31]. High concentrations of Firmicutes have been related with greater metabolic degradation of energy sources, which results in an increase of caloric absorption and, consequently, more weight gain. It should be noted that our patients were still considered at the border between overweight and obese at the end of the study according to their average BMI $(\approx 30)$. In this sense, increased Firmicutes abundance has been consistently associated to obesity [32], so promoting a change towards a healthy profile (lower Firmicutes) may be a determinant of the beneficial effects of bariatric surgery. In contrast, the Proteobacteria phylum has been associated with a beneficial profile characterized by decreased systemic inflammation and improved glucose homeostasis [7], and correlated with weight loss [28]. Moreover, the increase in luminal $\mathrm{pH}$ due to the reduction in gastric volume following bariatric surgery may be one of the factors that allow the proliferation of this taxa [33].

Surgical intervention also modified gut microbiota composition at the class level, increasing the abundance of Enterobacteriaceae, which has been negatively correlated with cholesterol levels in humans $[34,35]$ as well as positively correlated with weight loss in animal models [36], and whose increment has been previously reported after gastric bypass [37] and bilio-intestinal bypass [38]. Additionally, previous studies have postulated the reduction in the abundance of the Clostridiaceae family after bariatric surgery [36,38], which agrees with our results. On the contrary, the abundance of the Lachnospiraceae family members has been positively correlated with BMI, suggesting that the decreased abundance of this family in our study may be related to weight loss [39]. Surgical intervention also had a profound impact at the genus level. Thus, the decrease observed in the Coprococcus genus was previously found after different types of bariatric surgery [27,30], whereas the decreases observed in the Lactococcus genus were less consistent [27]. Nevertheless, high levels of Lactococcus have been associated with obesity and fasting plasma insulin [40]. Moreover, Acinetobacter overgrowth was present in patients under failed bariatric surgery [41], and in our study was positively correlated with LDL plasma concentration, so the significant reduction of this genus could indicate a more successful treatment. On the other hand, Faria et al. [42] reported that patients without weight regain after bariatric surgery presented an increased Phascolarctobacterium genus [42]. This finding does not agree with our results, because our patients showed a reduction in this genus, as well as a significant and persistent decrease in body weight four years after bariatric surgery. In contrast, bariatric surgery induced an increase in Butyricimonas, Parabacteroides and Slackia genera. Parabacteroides has been negatively correlated with serum insulin concentration after bariatric surgery [27] and with BMI in our study, whereas high levels of Slackia have been detected in patients after Roux-en-Y gastric bypass [35]. Furthermore, increased abundance of Butyricimonas has been associated with less food addiction after bariatric 
surgery [29]. Finally, Lachnospira decreased significantly in operated patients and was positively correlated with BMI, body fat and insulin levels, unlike a previous study in which Lachnospira was negatively correlated with BMI and fasting blood glucose [43]. Moreover, this genus correlated positively with TMAO and decreased with bariatric surgery, which is in accordance with its reported ability to produce this metabolite [44]. Furthermore, a recent study of the alterations on microbiota in childhood obesity showed that Lachnospira was significantly elevated in patients with obesity [45].

The faecal metabolome of the patients with severe obesity was also modified due to bariatric surgery, in accordance with previous findings [30,46], showing a different profile after four years of surgical intervention. In fact, methyl acetoacetate, carbamoyl aspartate and serine phosphate increased, whereas metabolites such as 5-aminolevulinic acid, choline, citric acid, malic acid, taurine, TMAO and tropic acid decreased in faeces, suggesting that long-term effects of surgical intervention affected this characteristic metabolomic profile. In this sense, previous studies described a reduction in choline [47,48], TMAO [48,49], taurine [50] or citric acid [49] with bariatric surgery, which agree with our results. Moreover, taurine was positively correlated with insulin and AST levels, whereas citric acid was correlated with the parameters AST, ALT and BMI. In this sense, taurine is an essential metabolite in bile acid formation, and high levels have been associated with obesity [51]. Related to the TMAO metabolite, this is derived from gut microbiota choline metabolism, whose high levels have been associated with organ damage and cardiovascular disease [49]. Although an increased concentration of this metabolite has been reported one year after bariatric surgery [48], our results showed that four years after intervention levels of TMAO were reduced, so this may be considered a long-term beneficial effect of bariatric surgery. Additionally, TMAO positively correlated with the presence of Lactococcus genus, which agrees with its role as a minor producer of this metabolite [44].

Moreover, exploring metabolomic pathways linked with metabolites that could be influenced by bariatric surgery, surgical intervention reduced the tricarboxylic acid cycle, glycine, serine and threonine metabolism, glyoxylate and dicarboxylate metabolism and tyrosine metabolism. These results suggest that branched-chain amino acids (BCAAs) and aromatic amino acids, as well as energetic metabolism, were downregulated with bariatric surgery, findings that have previously reported [49,52-55]. The reduction in BCAA levels as a consequence of bariatric surgery could be a normalization of the altered amino acid profile associated with obesity $[49,52,53]$, which has been linked to an impairment of glucose homeostasis [48,49]. Furthermore, citric or malic acid were reduced after bariatric surgery, which was directly linked with the observed downregulation in energetic metabolism, and could be a consequence of the decreasing glucose input and increasing gluconeogenesis [53].

SCFAs are metabolites produced by gut microbiota fermentation from carbohydrates involved in various physiological process, such as lipogenesis de novo and gluconeogenesis or energy harvest [56]. The role of SCFAs in health and disease is still controversial and is not completely understood. Therefore, high levels of these metabolites have been related to cardiometabolic and hepatic health [56-58], but also have been associated with gut dysbiosis, gut permeability and excess adiposity $[10,59]$. Moreover, elevated concentrations of the main SCFAs have been linked with obesity development and with a major energy extraction from the diet $[9,10,59]$. In our study, the SCFA faecal profiles of the patients after bariatric surgery were modified, with significant decreases of acetate, butyrate and propionate concentrations, in accordance with previous reports $[38,60]$ and with the reduction in the aforementioned energetic metabolic pathway. These SCFAs all positively correlated with BMI, reinforcing their reported role in obesity. Moreover, acetate, butyrate and propionate had a negative correlation with Butyricimonas and Parabacteroides genera, and a positive correlation with the Lachnospira genus. Although Butyricimonas is a butyrate producer, and this metabolite decreased in our study while the bacteria increased, there are many other bacteria involved in this process, which could explain these results. Additionally, the reduction in the Lachnospira genus agrees with the decrease in acetate, due to 
its role as a producer of this metabolite [61], and may represent a characteristic feature of weight loss because reduced abundance of SCFA producers (including Lachnospira genus) has also been reported in mildly obese patients under restrictive diets [39]. Furthermore, these changes could be attributed not only to a modification in gut microbiota but also to the reshaped metabolome after bariatric surgery. Altogether, correlation analysis showed a metagenomic and metabolomic profile related to bariatric surgery that could be involved in the beneficial effects observed on biochemical and anthropometric parameters.

This study presents some limitations. First, the difficulties in the collection of stool samples linked with the scarce number of patients. Second, male and female numbers were not equivalent. Although our results agree with current research, further studies with larger cohorts would be necessary to obtain more statistical power and to settle the relationship between gut microbiota and bariatric surgery. Another limitation is related to the surgical procedures, as more restrictive techniques such as sleeve gastrectomy could have different effects on gut microbiota than procedures with a malabsorptive component, such as BPD or gastric bypass. Because our sample was small, we did not consider these potential differences. Finally, although the patients underwent nutritional education by a registered dietitian, they were not under any specific diet, so the changes in gut microbiota composition could also be influenced by interindividual diet patterns. Related to this, collecting additional information about the lifestyle of the patients during this period (exercise, use of drugs, etc) would have been useful to guarantee that the specific changes observed in gut microbiota were due to bariatric surgery. In this sense, it has been previously reported that both diet and exercise have profound modulatory effects on gut microbiota composition [62] and are key factors in the success of bariatric surgery [63,64]. In fact, a higher protein diet has been shown to enhance satiety, body weight and fat loss in operated patients [64]. However, the consistency and homogeneity of our results across the individuals draws attention to a common cause of the modifications observed that should be attributed to bariatric surgery, independently of other source of interindividual discrepancies such as dietary patterns or exercise habits.

\section{Conclusions}

In conclusion, our findings point to bariatric surgery as a long-term modulator of gut microbiota, not only on its composition but also its functionality, promoting less efficient energy extraction from the diet as a possible mechanism linked to the persistent beneficial metabolic effects of a successful intervention. Nonetheless, more research and larger study populations are needed to determine the specific mechanisms by which the gut microbiota profile triggered by bariatric surgery is involved in the improvement of obesity.

Supplementary Materials: The following are available online at https:/ /www.mdpi.com/article/ 10.3390/nu13082519/s1. Figure S1: Effect of bariatric surgery on gut microbiota composition of patients with severe obesity. Figure S2: Effect of bariatric surgery on faecal metabolome of patients with severe obesity. Figure S3: Associations of gut microbiota profile with faecal short-chain fatty acids concentration.

Author Contributions: Conceptualization, E.N., F.J. and S.S.-C.; methodology, E.N. and F.J; formal analysis, E.N., S.S.-C.; investigation, M.J.-F., S.R.-S., D.P., M.V.G.-M., P.L., M.D.B.-P., A.U.-F., B.Á.-C., J.G.-G., S.S.-C., F.J. and E.N.; data curation, J.G.-G., S.S.-C., F.J. and E.N.; writing-original draft preparation, M.J.-F., D.P., M.V.G.-M., S.S.-C., F.J. and E.N.; writing-review and editing, M.J.-F., S.R.-S., D.P., M.V.G.-M., P.L., M.D.B.-P., A.U.-F., B.Á.-C., J.G.-G., S.S.-C., F.J. and E.N.; visualization, M.J.-F., S.R.-S., D.P., M.V.G.-M., P.L., M.D.B.-P., A.U.-F., B.Á.-C., J.G.-G., S.S.-C., F.J. and E.N.; supervision, J.G.-G., S.S.-C., F.J. and E.N.; project administration, J.G.-G., S.S.-C., F.J. and E.N.; funding acquisition, J.G.-G., S.S.-C. and F.J. All authors have read and agreed to the published version of the manuscript.

Funding: This research was funded by grants from Ministerio de Economía y Competitividad/FEDER (BFU2017-87960-R) and Junta de Castilla y León (GRS1888/A/18). 
Institutional Review Board Statement: The study was conducted according to the guidelines of the Declaration of Helsinki and approved by the Ethics Comitte of the Complejo Asistencial Universitario de León (CAULE) (protocol code 2135, 23 February 2021).

Informed Consent Statement: Informed consent was obtained from all subjects involved in the study. Written informed consent was obtained from the patients to publish this paper.

Data Availability Statement: The data presented in this study are available on request from the corresponding author.

Acknowledgments: CIBERehd is funded by the Instituto de Salud Carlos III, Spain. M.J.-F. was supported by a fellowship from Ministerio de Ciencia, Innovación y Universidades (FPU18/06257); S.R.-S. and D.P. by Junta de Castilla y León cofunded by the European Social Fund; and E.N. by CIBERehd contracts.

Conflicts of Interest: The authors declare no conflict of interest. The funders had no role in the design of the study, in the collection, analyses or interpretation of data, in the writing of the manuscript or in the decision to publish the results.

\section{References}

1. De Lorenzo, A.; Romano, L.; Di Renzo, L.; Di Lorenzo, N.; Cenname, G.; Gualtieri, P. Obesity: A preventable, treatable, but relapsing disease. Nutrition 2020, 71, 110615. [CrossRef]

2. Semlitsch, T.; Stigler, F.L.; Jeitler, K.; Horvath, K.; Siebenhofer, A. Management of overweight and obesity in primary care-A systematic overview of international evidence-based guidelines. Obes. Rev. 2019, 20, 1218-1230. [CrossRef] [PubMed]

3. Park, C.H.; Nam, S.J.; Choi, H.S.; Kim, K.O.; Kim, D.H.; Kim, J.W.; Sohn, W.; Yoon, J.H.; Jung, S.H.; Hyun, Y.S.; et al. Comparative efficacy of bariatric surgery in the treatment of morbid obesity and diabetes mellitus: A systematic review and network meta-analysis. Obes. Surg. 2019, 29, 2180-2190. [CrossRef] [PubMed]

4. Jiménez, A.; Ibarzabal, A.; Moizé, V.; Pané, A.; Andreu, A.; Molero, J.; de Hollanda, A.; Flores, L.; Ortega, E.; Lacy, A.; et al. Ten-year outcomes after Roux-en-Y gastric bypass and sleeve gastrectomy: An observational nonrandomized cohort study. Surg. Obes. Relat. Dis. 2019, 15, 382-388. [CrossRef] [PubMed]

5. Arterburn, D.E.; Telem, D.A.; Kushner, R.F.; Courcoulas, A.P. Benefits and risks of bariatric surgery in adults: A review. JAMA 2020, 324, 879-887. [CrossRef]

6. Cuevas-Sierra, A.; Ramos-Lopez, O.; Riezu-Boj, J.I.; Milagro, F.I.; Martinez, J.A. Diet, gut microbiota, and obesity: Links with host genetics and epigenetics and potential applications. Adv. Nutr. 2019, 10, S17-S30. [CrossRef]

7. Debédat, J.; Clément, K.; Aron-Wisnewsky, J. Gut microbiota dysbiosis in human obesity: Impact of bariatric surgery. Curr. Obes. Rep. 2019, 8, 229-242. [CrossRef]

8. Tremaroli, V.; Karlsson, F.; Werling, M.; Ståhlman, M.; Kovatcheva-Datchary, P.; Olbers, T.; Fändriks, L.; Le Roux, C.W.; Nielsen, J.; Bäckhed, F. Roux-en-Y gastric bypass and vertical banded gastroplasty induce long-term changes on the human gut microbiome contributing to fat mass regulation. Cell Metab. 2015, 22, 228-238. [CrossRef] [PubMed]

9. Turnbaugh, P.J.; Ley, R.E.; Mahowald, M.A.; Magrini, V.; Mardis, E.R.; Gordon, J.I. An obesity-associated gut microbiome with increased capacity for energy harvest. Nature 2006, 444, 1027-1031. [CrossRef]

10. de la Cuesta-Zuluaga, J.; Mueller, N.T.; Álvarez-Quintero, R.; Velásquez-Mejía, E.P.; Sierra, J.A.; Corrales-Agudelo, V.; Carmona, J.A.; Abad, J.M.; Escobar, J.S. Higher fecal short-chain fatty acid levels are associated with gut microbiome dysbiosis, obesity, hypertension and cardiometabolic disease risk factors. Nutrients 2019, 11, 51. [CrossRef]

11. Tilg, H.; Zmora, N.; Adolph, T.E.; Elinav, E. The intestinal microbiota fuelling metabolic inflammation. Nat. Rev. Immunol. 2020, 20, 40-54. [CrossRef]

12. Rinninella, E.; Cintoni, M.; Raoul, P.; Ianiro, G.; Laterza, L.; Lopetuso, L.R.; Ponziani, F.R.; Gasbarrini, A.; Mele, M.C. Gut microbiota during dietary restrictions: New insights in non-communicable diseases. Microorganisms 2020, 8, 1140. [CrossRef]

13. Fabbiano, S.; Suárez-Zamorano, N.; Chevalier, C.; Lazarević, V.; Kieser, S.; Rigo, D.; Leo, S.; Veyrat-Durebex, C.; Gaïa, N.; Maresca, M.; et al. Functional gut microbiota remodeling contributes to the caloric restriction-induced metabolic improvements. Cell Metab. 2018, 28, 907-921.e7. [CrossRef] [PubMed]

14. Heianza, Y.; Sun, D.; Smith, S.R.; Bray, G.A.; Sacks, F.M.; Qi, L. Changes in gut microbiota-related metabolites and longterm successful weight loss in response to weight-loss diets: The POUNDS lost trial. Diabetes Care 2018, 41, 413-419. [CrossRef]

15. Porras, D.; Nistal, E.; Martínez-Flórez, S.; Pisonero-Vaquero, S.; Olcoz, J.L.; Jover, R.; González-Gallego, J.; García-Mediavilla, M.V.; Sánchez-Campos, S. Protective effect of quercetin on high-fat diet-induced non-alcoholic fatty liver disease in mice is mediated by modulating intestinal microbiota imbalance and related gut-liver axis activation. Free Radic. Biol. Med. 2017, 102, 188-202. [CrossRef] [PubMed]

16. Porras, D.; Nistal, E.; Martínez-Flórez, S.; Olcoz, J.L.; Jover, R.; Jorquera, F.; González-Gallego, J.; García-Mediavilla, M.V.; Sánchez-Campos, S. Functional interactions between gut microbiota transplantation, quercetin, and high-fat diet determine non-alcoholic fatty liver disease development in germ-free mice. Mol. Nutr. Food Res. 2019, 63, e1800930. [CrossRef]

17. Babraham Bioinformatics. Available online: http://www.bioinformatics.babraham.ac.uk (accessed on 20 May 2020). 
18. Caporaso, J.G.; Kuczynski, J.; Stombaugh, J.; Bittinger, K.; Bushman, F.D.; Costello, E.K.; Fierer, N.; Pẽa, A.G.; Goodrich, J.K.; Gordon, J.I.; et al. QIIME allows analysis of high-throughput community sequencing data. Nat. Methods 2010, 7, 335-336. [CrossRef]

19. Edgar, R.C.; Haas, B.J.; Clemente, J.C.; Quince, C.; Knight, R. UCHIME improves sensitivity and speed of chimera detection. Bioinformatics 2011, 27, 2194-2200. [CrossRef] [PubMed]

20. Edgar, R.C. Search and clustering orders of magnitude faster than BLAST. Bioinformatics 2010, 26, 2460-2461. [CrossRef] [PubMed]

21. Caporaso, J.G.; Bittinger, K.; Bushman, F.D.; Desantis, T.Z.; Andersen, G.L.; Knight, R. PyNAST: A flexible tool for aligning sequences to a template alignment. Bioinformatics 2010, 26, 266-267. [CrossRef] [PubMed]

22. Johnsen, L.G.; Skou, P.B.; Khakimov, B.; Bro, R. Gas chromatography-Mass spectrometry data processing made easy. J. Chromatogr. A 2017, 1503, 57-64. [CrossRef]

23. Aron-Wisnewsky, J.; Prifti, E.; Belda, E.; Ichou, F.; Kayser, B.D.; Dao, M.C.; Verger, E.O.; Hedjazi, L.; Bouillot, J.L.; Chevallier, J.M.; et al. Major microbiota dysbiosis in severe obesity: Fate after bariatric surgery. Gut 2019, 68, 70-82. [CrossRef]

24. Ballesteros Pomar, M.D.; Vilarrasa García, N.; Rubio Herrera, M.Á.; Barahona, M.J.; Bueno, M.; Caixàs, A.; Calañas Continente, A.; Ciudin, A.; Cordido, F.; de Hollanda, A.; et al. The SEEN comprehensive clinical survey of adult obesity: Executive summary. Endocrinol. Diabetes Nutr. 2021, 68, 130-136. [CrossRef] [PubMed]

25. Cooper, T.C.; Simmons, E.B.; Webb, K.; Burns, J.L.; Kushner, R.F. Trends in weight regain following Roux-en-Y Gastric Bypass (RYGB) bariatric surgery. Obes. Surg. 2015, 25, 1474-1481. [CrossRef]

26. Koulas, S.G.; Stefanou, C.K.; Stefanou, S.K.; Tepelenis, K.; Zikos, N.; Tepetes, K.; Kapsoritakis, A. Gut microbiota in patients with morbid obesity before and after bariatric surgery: A ten-year review study (2009-2019). Obes. Surg. 2021, 31, 317-326. [CrossRef]

27. Guo, Y.; Huang, Z.P.; Liu, C.Q.; Qi, L.; Sheng, Y.; Zou, D.J. Modulation of the gut microbiome: A systematic review of the effect of bariatric surgery. Eur. J. Endocrinol. 2018, 178, 43-56. [CrossRef]

28. Luijten, J.C.H.B.M.; Vugts, G.; Nieuwenhuijzen, G.A.P.; Luyer, M.D.P. The importance of the microbiome in bariatric surgery: A systematic review. Obes. Surg. 2019, 29, 2338-2349. [CrossRef] [PubMed]

29. Oduro-Donkor, D.; Turner, M.C.; Farnaud, S.; Renshaw, D.; Kyrou, I.; Hanson, P.; Hattersley, J.; Weickert, M.O.; Menon, V.; Randeva, H.S.; et al. Modification of fecal microbiota as a mediator of effective weight loss and metabolic benefits following bariatric surgery. Expert Rev. Endocrinol. Metab. 2020, 15, 363-373. [CrossRef] [PubMed]

30. Yu, D.; Shu, X.O.; Howard, E.F.; Long, J.; English, W.J.; Flynn, C.R. Fecal metagenomics and metabolomics reveal gut microbial changes after bariatric surgery. Surg. Obes. Relat. Dis. 2020, 16, 1772-1782. [CrossRef]

31. Shen, N.; Caixàs, A.; Ahlers, M.; Patel, K.; Gao, Z.; Dutia, R.; Blaser, M.J.; Clemente, J.C.; Laferrère, B. Longitudinal changes of microbiome composition and microbial metabolomics after surgical weight loss in individuals with obesity. Surg. Obes. Relat. Dis. 2019, 15, 1367-1373. [CrossRef] [PubMed]

32. Crovesy, L.; Masterson, D.; Lopes Rosado, E. Profile of the gut microbiota of adults with obesity: A systematic review. Eur. J. Clin. Nutr. 2020, 74, 1251-1262. [CrossRef]

33. Ciobârcă, D.; Cătoi, A.F.; Copăescu, C.; Miere, D.; Crișan, G. Bariatric surgery in obesity: Effects on gut microbiota and micronutrient status. Nutrients 2020, 12, 235. [CrossRef]

34. Paganelli, F.L.; Luyer, M.; Hazelbag, C.M.; Uh, H.W.; Rogers, M.R.C.; Adriaans, D.; Berbers, R.M.; Hendrickx, A.P.A.; Viveen, M.C.; Groot, J.A.; et al. Roux-Y Gastric Bypass and sleeve gastrectomy directly change gut microbiota composition independent of surgery type. Sci. Rep. 2019, 9, 10979. [CrossRef]

35. Sánchez-Alcoholado, L.; Gutiérrez-Repiso, C.; Gómez-Pérez, A.M.; García-Fuentes, E.; Tinahones, F.J.; Moreno-Indias, I. Gut microbiota adaptation after weight loss by Roux-en-Y gastric bypass or sleeve gastrectomy bariatric surgeries. Surg. Obes. Relat. Dis. 2019, 15, 1888-1895. [CrossRef] [PubMed]

36. Li, J.V.; Ashrafian, H.; Bueter, M.; Kinross, J.; Sands, C.; Le Roux, C.W.; Bloom, S.R.; Darzi, A.; Athanasiou, T.; Marchesi, J.R.; et al. Metabolic surgery profoundly influences gut microbial-Host metabolic cross-talk. Gut 2011, 60, 1214-1223. [CrossRef]

37. Zhang, H.; DiBaise, J.K.; Zuccolo, A.; Kudrna, D.; Braidotti, M.; Yu, Y.; Parameswaran, P.; Crowell, M.D.; Wing, R.; Rittmann, B.E.; et al. Human gut microbiota in obesity and after gastric bypass. Proc. Natl. Acad. Sci. USA 2009, 106, 2365-2370. [CrossRef] [PubMed]

38. Patrone, V.; Vajana, E.; Minuti, A.; Callegari, M.L.; Federico, A.; Loguercio, C.; Dallio, M.; Tolone, S.; Docimo, L.; Morelli, L. Postoperative changes in fecal bacterial communities and fermentation products in obese patients undergoing bilio-intestinal bypass. Front. Microbiol. 2016, 7, 200. [CrossRef] [PubMed]

39. Seganfredo, F.; Blume, C.; Moehlecke, M.; Giongo, A.; Casagrande, D.; Spolidoro, J.; Padoin, A.; Schaan, B.; Mottin, C. Weight-loss interventions and gut microbiota changes in overweight and obese patients: A systematic review. Obes. Rev. 2017, 18, 832-851. [CrossRef] [PubMed]

40. Qiao, Y.; Sun, J.; Xie, Z.; Shi, Y.; Le, G. Propensity to high-fat diet-induced obesity in mice is associated with the indigenous opportunistic bacteria on the interior of Peyer's patches. J. Clin. Biochem. Nutr. 2014, 55, 120-128. [CrossRef]

41. Gutiérrez-Repiso, C.; Moreno-Indias, I.; de Hollanda, A.; Martín-Núñez, G.M.; Vidal, J.; Tinahones, F.J. Gut microbiota specific signatures are related to the successful rate of bariatric surgery. Am. J. Transl. Res. 2019, 11, 942-952.

42. Faria, S.L.; Santos, A.; Magro, D.O.; Cazzo, E.; Assalin, H.B.; Guadagnini, D.; Vieira, F.T.; Dutra, E.S.; Saad, M.J.A.; Ito, M.K. Gut microbiota modifications and weight regain in morbidly obese women after Roux-en-Y gastric bypass. Obes. Surg. 2020, 30, 4958-4966. [CrossRef] [PubMed] 
43. Zhang, Q.; Xiao, X.; Li, M.; Yu, M.; Ping, F.; Zheng, J.; Wang, T.; Wang, X. Vildagliptin increases butyrate-producing bacteria in the gut of diabetic rats. PLOS ONE 2017, 12, e0184735. [CrossRef]

44. Cho, C.E.; Aardema, N.D.J.; Bunnell, M.L.; Larson, D.P.; Aguilar, S.S.; Bergeson, J.R.; Malysheva, O.V.; Caudill, M.A.; Lefevre, M. Effect of choline forms and gut microbiota composition on trimethylamine-n-oxide response in healthy men. Nutrients 2020, 12, 2220. [CrossRef] [PubMed]

45. Chen, X.; Sun, H.; Jiang, F.; Shen, Y.; Li, X.; Hu, X.; Shen, X.; Wei, P. Alteration of the gut microbiota associated with childhood obesity by 16S rRNA gene sequencing. PeerJ 2020, 8, e8317. [CrossRef] [PubMed]

46. Sanchez-Carrillo, S.; Ciordia, S.; Rojo, D.; Zubeldia-Varela, E.; Méndez-García, C.; Martínez-Martínez, M.; Barbas, C.; Ruiz-Ruiz, S.; Moya, A.; Garriga, M.; et al. A body weight loss- and health-promoting gut microbiota is established after bariatric surgery in individuals with severe obesity. J. Pharm. Biomed. Anal. 2021, 193, 113747. [CrossRef] [PubMed]

47. Yoshida, N.; Kitahama, S.; Yamashita, T.; Hirono, Y.; Tabata, T.; Saito, Y.; Shinohara, R.; Nakashima, H.; Emoto, T.; Hirota, Y.; et al. Metabolic alterations in plasma after laparoscopic sleeve gastrectomy. J. Diabetes Investig. 2021, 12, 123-129. [CrossRef]

48. Narath, S.H.; Mautner, S.I.; Svehlikova, E.; Schultes, B.; Pieber, T.R.; Sinner, F.M.; Gander, E.; Libiseller, G.; Schimek, M.G.; Sourij, $\mathrm{H}$; et al. An untargeted metabolomics approach to characterize short-term and long-term metabolic changes after bariatric surgery. PLoS ONE 2016, 11, 1-18. [CrossRef]

49. Gralka, E.; Luchinat, C.; Tenori, L.; Ernst, B.; Thurnheer, M.; Schultes, B. Metabolomic fingerprint of severe obesity is dynamically affected by bariatric surgery in a procedure-dependent manner. Am. J. Clin. Nutr. 2015, 102, 1313-1322. [CrossRef]

50. Ilhan, Z.E.; DiBaise, J.K.; Dautel, S.E.; Isern, N.G.; Kim, Y.M.; Hoyt, D.W.; Schepmoes, A.A.; Brewer, H.M.; Weitz, K.K.; Metz, T.O.; et al. Temporospatial shifts in the human gut microbiome and metabolome after gastric bypass surgery. NPJ Biofilms Microbiomes 2020, 6, 1-12. [CrossRef]

51. Mediani, A.; Abas, F.; Maulidiani, M.; Khatib, A.; Tan, C.P.; Ismail, I.S.; Shaari, K.; Ismail, A.; Lajis, N.H. Metabolic and biochemical changes in streptozotocin induced obese-diabetic rats treated with Phyllanthus niruri extract. J. Pharm. Biomed. Anal. 2016, 128, 302-312. [CrossRef]

52. Lopes, T.I.B.; Geloneze, B.; Pareja, J.C.; Calixto, A.R.; Ferreira, M.M.C.; Marsaioli, A.J. Omics prospective monitoring of bariatric surgery: Roux-en-Y gastric bypass outcomes using mixed-meal tolerance test and time-resolved 1H NMR-based metabolomics. Omi. A J. Integr. Biol. 2016, 20, 415-423. [CrossRef] [PubMed]

53. Lopes, T.I.B.; Geloneze, B.; Pareja, J.C.; Calixto, A.R.; Ferreira, M.M.C.; Marsaioli, A.J. Blood metabolome changes before and after bariatric surgery: A 1H NMR-based clinical investigation. Omi. A J. Integr. Biol. 2015, 19, 318-327. [CrossRef] [PubMed]

54. Palau-Rodriguez, M.; Tulipani, S.; Marco-Ramell, A.; Miñarro, A.; Jáuregui, O.; Sanchez-Pla, A.; Ramos-Molina, B.; Tinahones, F.J.; Andres-Lacueva, C. Metabotypes of response to bariatric surgery independent of the magnitude of weight loss. PLoS ONE 2018, 13, e0198214. [CrossRef]

55. Wijayatunga, N.N.; Sams, V.G.; Dawson, J.A.; Mancini, M.L.; Mancini, G.J.; Moustaid-Moussa, N. Roux-en-Y gastric bypass surgery alters serum metabolites and fatty acids in patients with morbid obesity. Diabetes. Metab. Res. Rev. 2018, 34, e3045. [CrossRef] [PubMed]

56. Kasubuchi, M.; Hasegawa, S.; Hiramatsu, T.; Ichimura, A.; Kimura, I. Dietary gut microbial metabolites, short-chain fatty acids, and host metabolic regulation. Nutrients 2015, 7, 2839-2849. [CrossRef]

57. Quiroga, R.; Nistal, E.; Estébanez, B.; Porras, D.; Juárez-Fernández, M.; Martínez-Flórez, S.; García-Mediavilla, M.V.; de Paz, J.A.; González-Gallego, J.; Sánchez-Campos, S.; et al. Exercise training modulates the gut microbiota profile and impairs inflammatory signaling pathways in obese children. Exp. Mol. Med. 2020, 52, 1048-1061. [CrossRef]

58. Carbajo-Pescador, S.; Porras, D.; Garcia-Mediavilla, M.V.; Martinez-Florez, S.; Juarez-Fernandez, M.; Cuevas, M.J.; Mauriz, J.L.; Gonzalez-Gallego, J.; Nistal, E.; Sanchez-Campos, S. Beneficial effects of exercise on gut microbiota functionality and barrier integrity, and gut-liver crosstalk in an in vivo model of early obesity and non-alcoholic fatty liver disease. DMM Dis. Model. Mech. 2019, 12, dmm039206. [CrossRef]

59. Kim, K.N.; Yao, Y.; Ju, S.Y. Short chain fatty acids and fecal microbiota abundance in humans with obesity: A systematic review and meta-analysis. Nutrients 2019, 11, 2512. [CrossRef]

60. Liou, A.P.; Paziuk, M.; Luevano, J.M.; Machineni, S.; Turnbaugh, P.J.; Kaplan, L.M. Conserved shifts in the gut microbiota due to gastric bypass reduce host weight and adiposity. Sci. Transl. Med. 2013, 5, 178ra41. [CrossRef]

61. Mayengbam, S.; Lambert, J.E.; Parnell, J.A.; Tunnicliffe, J.M.; Nicolucci, A.C.; Han, J.; Sturzenegger, T.; Shearer, J.; Mickiewicz, B.; Vogel, H.J.; et al. Impact of dietary fiber supplementation on modulating microbiota-host-metabolic axes in obesity. J. Nutr. Biochem. 2019, 64, 228-236. [CrossRef]

62. Juárez-Fernández, M.; Porras, D.; García-Mediavilla, M.V.; Román-Sagüillo, S.; González-Gallego, J.; Nistal, E.; Sánchez-Campos, S. Aging, gut microbiota and metabolic diseases: Management through physical exercise and nutritional interventions. Nutrients 2021, 13, 16. [CrossRef]

63. Hatoum, I.J.; Stein, H.K.; Merrifield, B.F.; Kaplan, L.M. Capacity for physical activity predicts weight loss after roux-en-Y gastric bypass. Obesity 2009, 17, 92-99. [CrossRef] [PubMed]

64. Tabesh, M.R.; Maleklou, F.; Ejtehadi, F.; Alizadeh, Z. Nutrition, physical activity, and prescription of supplements in pre- and post-bariatric surgery patients: A practical guideline. Obes. Surg. 2019, 29, 3385-3400. [CrossRef] [PubMed] 